Research Article

\title{
Cross-Linked Chitosan as an Eco-Friendly Binder for High- Performance Wood-Based Fiberboard
}

\author{
Erzhuo Huang, ${ }^{1,2}$ Yanwei Cao, ${ }^{1}$ Xinpeng Duan, ${ }^{1}$ Yutao Yan, ${ }^{1}$ Zhe Wang $₫,{ }^{1,2}$ \\ and Chunde Jin (iD) 1 \\ ${ }^{1}$ School of Engineering, Zhejiang A \& F University, Hangzhou 311300, China \\ ${ }^{2}$ National Forestry and Grassland Administration Key Laboratory of Plant Fiber Functional Materials, Fuzhou, \\ Fujian 350002, China
}

Correspondence should be addressed to Zhe Wang; wangzhe@zafu.edu.cn and Chunde Jin; jincd@zafu.edu.cn Received 30 April 2021; Accepted 9 June 2021; Published 2 July 2021

Academic Editor: Jingpeng Li

Copyright (C) 2021 Erzhuo Huang et al. This is an open access article distributed under the Creative Commons Attribution License, which permits unrestricted use, distribution, and reproduction in any medium, provided the original work is properly cited.

\begin{abstract}
High-performance wood-based fiberboards with high strength and dimensional stability were fabricated by hot-pressing method using 2,5-dimethoxy-2,5-dihydrofuran (DHF) cross-linked chitosan (CS) as an eco-friendly binder. The effects of cross-linked chitosan on the mechanical properties and dimensional stability of wood-based fiberboards were investigated. It is evident that cross-linked chitosan addition was effective in improving mechanical properties and dimensional stability of wood-based fiberboards. The prepared wood-based fiberboard bonded by DHF cross-linked CS displayed optimum modulus of rupture (MOR) of $42.1 \mathrm{MPa}$, modulus of elasticity (MOE) of $3986.0 \mathrm{MPa}$, internal bonding (IB) strength of $1.4 \mathrm{MPa}$, and thickness swelling (TS) value of $16.3 \%$. The improvement of physical and mechanical properties of wood-based fiberboards could be attributed to the amide linkages and hydrogen bonds between wood fibers and cross-linked chitosan. The high-performance wood-based fiberboards fabricated in this study may be a promising candidate for eco-friendly wood-based composites.
\end{abstract}

\section{Introduction}

Wood-based fiberboard as a conventional composite has been widely used for many applications in our daily life, for instance, interior flooring, home, and office furniture [1]. In general, the mechanical strength of wood-based fiberboard relies heavily on the use of adhesives. Formaldehyde-based adhesives are most commonly used because of their good adhesive performance and relatively low cost. However, the release of free formaldehyde which poses a threat to human health and living environment during manufacture and use of wood-based fiberboard has attracted strong attention. Meanwhile, nonrenewable and increasingly depleted petroleum resources are needed for formaldehyde production. To resolve these issues, the traditional formaldehyde-based adhesives have been gradually replaced by bio-based adhesives with renewability and environmental friendliness or modified adhesives with formaldehyde scavengers. Further- more, isocyanate adhesives without formaldehyde are also good candidates for manufacturing environmentally friendly fiberboards. Nevertheless, compared with the conventional formaldehyde-based adhesives, the higher price also hinders more extensive use of isocyanate adhesives for wood-based panel manufacture. Therefore, there is an insistent demand to develop a green fabrication process of fiberboards by using eco-friendly and renewable adhesives.

Formaldehyde-free bio-based adhesives with renewability have been tried to fabricate fiberboard [2], for instance, lignin and its derivatives derived from plants [3, 4], plant protein derived from soy and wheat [5-7], nanocellulose [8, 9], and starch $[10,11]$. Nevertheless, they have not been widely used in fiberboard manufacturing on account of their complicated preparation processes, relatively weak bonding performance, and water resisting property. Based on the above issues, there is an urgent need to develop green fiberboards with facile fabrication processes, high bonding 
strength, and good dimensional stability.

Chitosan with renewability and biodegradability, a product of natural polysaccharide chitin via removing part of acetyl group, has attracted an extensive attention because of their rich source [12]. It has been widely used in food additives, textile, agriculture, environmental protection, beauty care, cosmetics, antibacterial agent, medical fiber due to its many physiological functions, such as biodegradability, biocompatibility, nontoxicity, antibacterial, anticancer, lipidlowering, and immunological enhancement [13-15], and so on. Chitosan possesses a lot of free amino and hydroxyl groups on its framework, which can afford bonding strengths between materials [16, 17]. Recently, wood-based materials also achieve good bonding strengths by using chitosanbased adhesives [18-20]. However, the linear nature of chitosan structure could lead to the deformation of materials. To overcome this structural limitation, the three-dimensional polymer networks of chitosan have been created by the chemical cross-linking [21-23].

Herein, a high-performance wood-based fiberboard with eco-friendly cross-linked chitosan binder has been successfully prepared by a facile hot-pressing process. The fiberboard achieved excellent physical and mechanical properties due to the addition of cross-linked chitosan. The effects of 2,5dimethoxy-2,5-dihydrofuran cross-linking treatment on the physical and mechanical properties of the prepared woodbased fiberboard were studied. The as-prepared wood-based fiberboard with outstanding mechanical properties could be regarded as a promising candidate for the environmentally friendly decoration materials.

\section{Materials and Methods}

2.1. Materials. Poplar wood (Populus tomentosa Carr.) fibers (water content is about $10 \%$ ) were provided by Zhejiang New Wood Material Technology Co., Ltd. (Ningbo, China). Chitosan (CS) was purchased from Macklin Biochemical (Shanghai, China). Acetic acid was supplied by Lingfeng Chemical Reagent Co., Ltd. (Shanghai, China). 2,5-Dimethoxy-2,5-dihydrofuran (DHF) was provided by Sinopharm Chemical Reagent Co., Ltd. (Shanghai, China). All chemicals were used as received without any further purification.

2.2. Preparation of Cross-Linked Chitosan Binder. The crosslinked CS binder was prepared as follows: CS powder was solubilized in acid aqueous solution with glacial acetic acid $(1.3 \%, w / v)$ at room temperature (about $20^{\circ} \mathrm{C}$ ) under stirring, which formed a stable CS solution $(2 \%, w / v)$. Afterwards, DHF $(0.1 \mathrm{M})$ was dropwise injected into the CS solution under continuous agitation. Then, the mixed solution reacted at $70^{\circ} \mathrm{C}$ for about 40 minutes until the formation of the $\mathrm{DHF}$ cross-linked CS hydrogel.

2.3. Preparation of Wood-Based Fiberboard Bonded by DHF Cross-Linked CS (F-DHF-CS). The preparation process of wood-based fiberboard is shown in Figure 1. Fiberboard forming is achieved by hot-pressing process. Before hotpressing process, the wood fibers and DHF cross-linked CS hydrogel were mixed in a mass ratio of $4.5: 100$ (CS to absolute dry wood fibers). Afterwards, the blended fibers were according to the national standard of the People's Republic of China GB/T11718-2009 under $180^{\circ} \mathrm{C}$ temperature, 4.0 $\mathrm{MPa}$ pressure (unit pressure of fiberboard surface), and 9 min pressing time to form a board with a size of $200 \mathrm{~mm} \times$ $200 \mathrm{~mm} \times 3 \mathrm{~mm}$. The density of the fiberboard was $0.95 \pm$ $0.02 \mathrm{~g} / \mathrm{cm}^{3}$. The fiberboard with the same amounts of water (F-W), fiberboard with the same amounts of CS (F-CS), and fiberboard with the same amounts of DHF (F-DHF) as the control groups were prepared by the above same process.

2.4. Characterization. The morphologies of fiberboards were observed by scanning electron microscopy (SEM, TM3030, Hitachi). The chemical groups of fiberboards were recorded by Fourier transform infrared (FTIR) spectroscopy (iS10, Nicolet) via a $\mathrm{KBr}$ pellet pressing method. The surface composition of fiberboards was carried out on X-ray photoelectron spectroscopy (XPS, ESCALAB 250XI, Thermo Fisher). The mechanical properties of fiberboards were tested by a universal mechanical testing machine (5960, Instron) according to Chinese national standard (GB/T 176572013). The three-point bending tests with a loading speed of $5 \mathrm{~mm} \mathrm{~min}^{-1}$ were used to investigate the modulus of rupture (MOR) and modulus of elasticity (MOE) of fiberboards. The internal bonding (IB) strengths of fiberboard samples with a size of $50 \mathrm{~mm} \times 50 \mathrm{~mm} \times 3 \mathrm{~mm}$ were evaluated by vertical tensile test with a loading speed of $1.0 \mathrm{~mm} \mathrm{~min}^{-1}$. The dimensional stabilities of fiberboard samples were analyzed by the measurements of $24 \mathrm{~h}$ thickness swelling (TS).

\section{Results and Discussion}

The surface morphologies of different fiberboards (F-W, FDHF, F-CS, F-DHF-CS) from the top view are shown in Figure 2. It is obvious that fibers in different fiberboards show different states and morphologies. As shown in Figures 2(a) and 2(b), the fibers possessed more loose states and relatively smooth surface. After CS and DHF cross-linked CS were introduced, there was a noticeable change in the states of the fibers (Figures 2(c) and 2(d)). The surfaces of F-CS and F-DHF-CS displayed a rougher morphology after the additions of CS and DHF cross-linked CS hydrogel, indicating that the CS and DHF cross-linked CS were successfully attached to the fiber surfaces. Meanwhile, tighter structures were observed on account of the bonding effect of the CS and DHF cross-linked CS at the interface between the two fibers.

The cross-section morphologies of different fiberboards (F-W, F-DHF, F-CS, F-DHF-CS) are shown in Figure S1. As shown in Figure S1a and Figure S1b, the cross sections of the F-W and F-DHF were fluffy and detached from each other. In comparison, the F-CS and F-DHF-CS showed tighter layered structure with less voids. Therefore, the loose structures of F-W and F-DHF could result in their poor mechanical properties. The tight and dense structures of F-DHF-CS could be beneficial to the improvement of its mechanical property. 

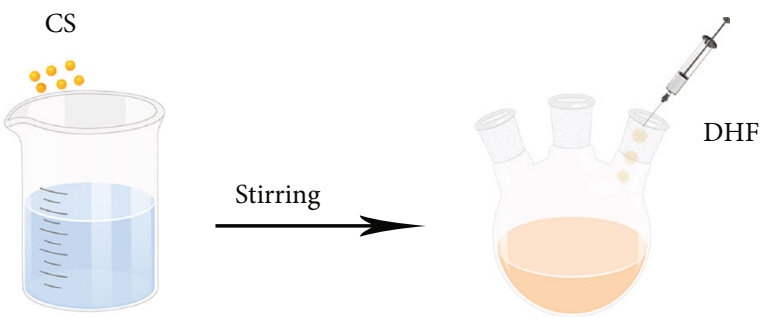

Acetic acid solution

$70{ }^{\circ} \mathrm{C}$ Stirring

Wood fiber

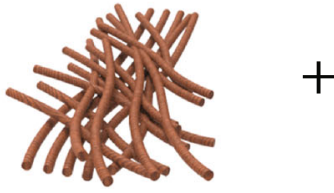

$+$

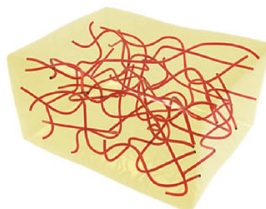

Crosslinked CS

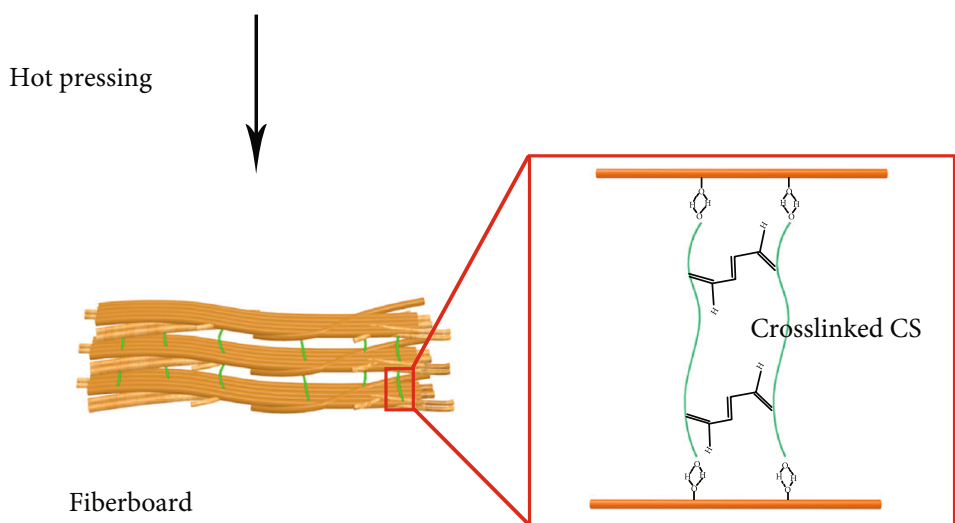

Figure 1: Schematic illustration of the fabrication of fiberboard bonded by DHF cross-linked CS.

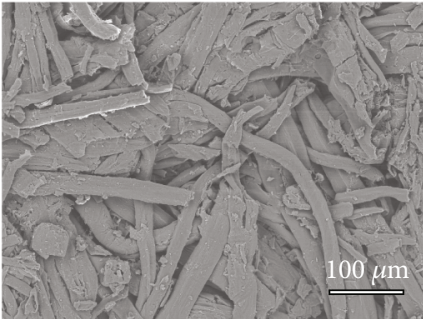

(a)

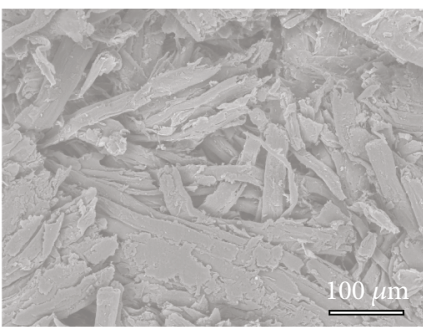

(c)

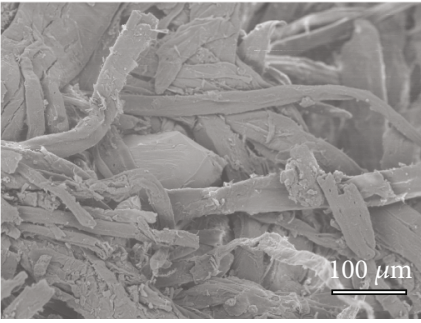

(b)

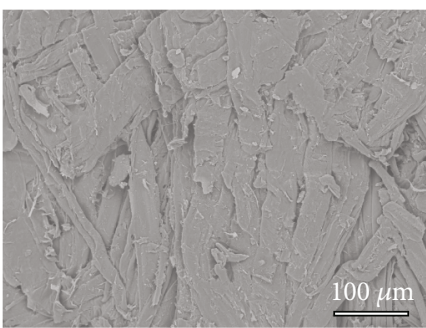

(d)

Figure 2: Top view SEM images: (a) F-W; (b) F-DHF; (c) F-CS; (d) F-DHF-CS. 


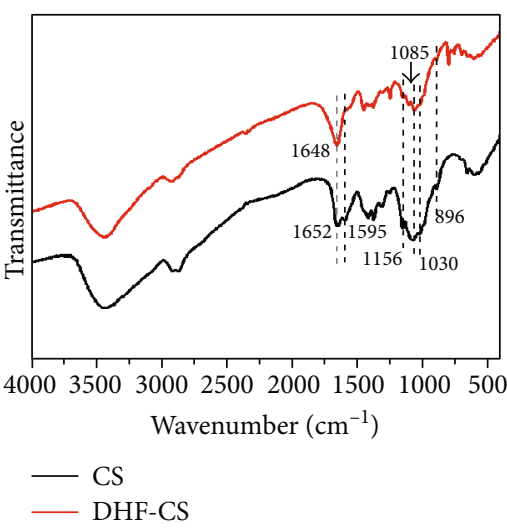

(a)

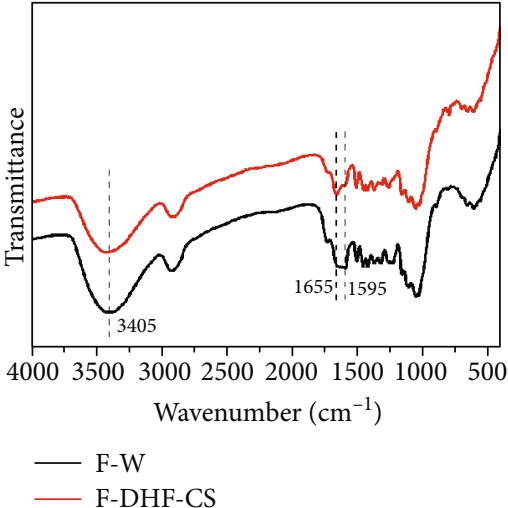

(b)

FIGURE 3: FTIR spectra of (a) CS and DHF cross-linked CS, (b) F-W, and F-DHF-CS.<smiles>COC1C=CC(OC)O1</smiles>

DHF

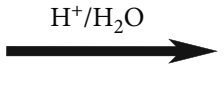

Malealdehyde

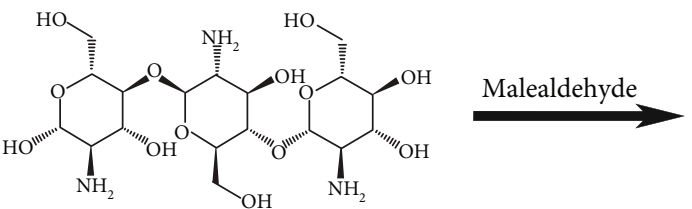

Chitosan

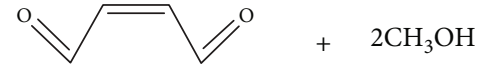

Malealdehyde

Figure 4: Chitosan cross-linked by the Schiff base reaction between malealdehyde and amine moieties of chitosan.

Figure S2 shows the XPS spectra of F-W, DHF crosslinked CS, and F-DHF-CS. As can be seen from Figure S2, F-W, DHF cross-linked CS, and F-DHF-CS displayed two main characteristic peaks at $285.1 \mathrm{eV}$ and $533.1 \mathrm{eV}$ which are attributed to the $\mathrm{C} 1 \mathrm{~s}$ and $\mathrm{O} 1 \mathrm{~s}$ [24-26], respectively. Compared with F-DHF-CS, DHF cross-linked CS has a more obvious characteristic peak at $399.1 \mathrm{eV}$. Compared with F-W, F-DHF-CS possessed a more obvious characteristic peak at $399.1 \mathrm{eV}$ which was corresponded to the N1s [27], indicating that wood fiber surface was successfully covered by the nitrogen-containing crosslinked CS.

Figure 3(a) shows the FTIR spectra of CS and DHF crosslinked CS. As can be seen from Figure 3(a), the CS and DHF cross-linked CS presented peaks at $1156 \mathrm{~cm}^{-1}, 1085 \mathrm{~cm}^{-1}$, $1030 \mathrm{~cm}^{-1}$, and $896 \mathrm{~cm}^{-1}$, which are attributed to glucosamine unit of chitosan [28]. The FTIR spectra of CS exhibited characteristic peaks of amide I $\left(1652 \mathrm{~cm}^{-1}\right)$ and amide II (1595 $\mathrm{cm}^{-1}$ ) [29]. DHF cross-linked CS presented an obvious characteristic peak at $1648 \mathrm{~cm}^{-1}$ attributed to the formation of $\mathrm{C}=\mathrm{N}$ of the Schiff's base structure due to the interaction between the glucosamine units of CS and the forming dialdehyde groups of DHF. Furthermore, DHF cross-linked CS showed a decreasing intensity of amine moieties at 1595 $\mathrm{cm}^{-1}$. This further indicated that the cross-linking reaction between CS and DHF occurred [30]. Schematic illustration of chitosan cross-linking reaction with DHF is depicted in Figure 4.

The FTIR spectra of F-W and F-DHF-CS are shown in Figure 3(b). The characteristic peak intensity of F-DHF-CS at $1655 \mathrm{~cm}^{-1}$ (amide linkages) was higher than that of F-W. This could be due to the reaction between the amine groups of cross-linked chitosan binder and carbonyl groups of wood fibers, which formed amide linkages. Moreover, the characteristic peak at $1595 \mathrm{~cm}^{-1}$ which was assigned to the superposition of the $\mathrm{C}=\mathrm{C}$ linkages of lignin and the $\mathrm{C}=\mathrm{O}$ amide 


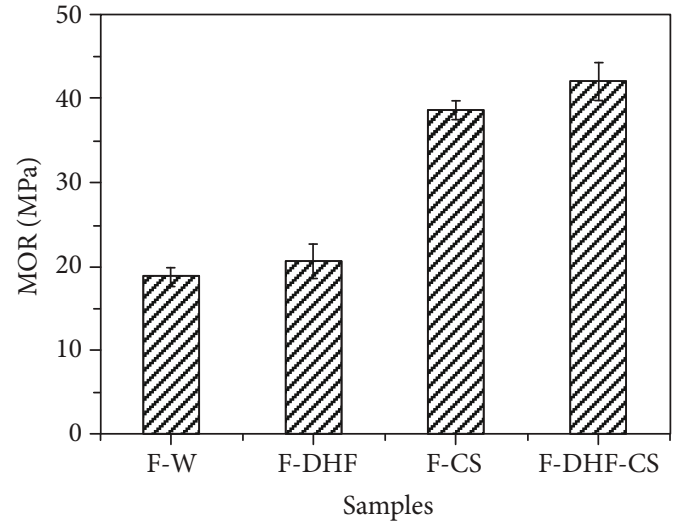

(a)

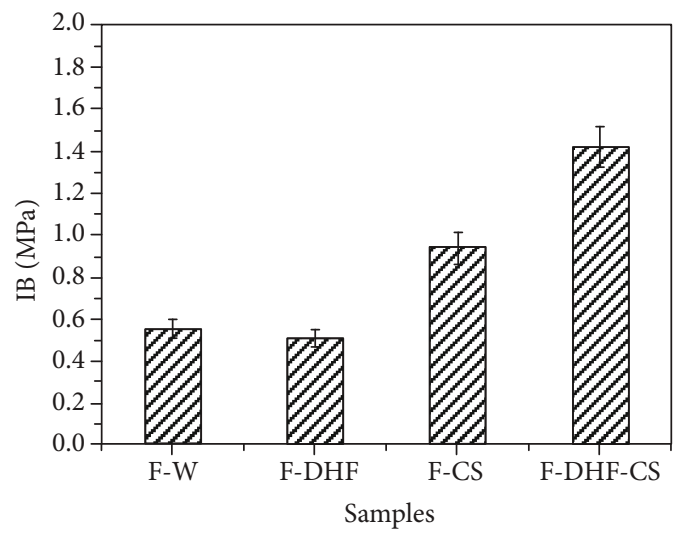

(c)

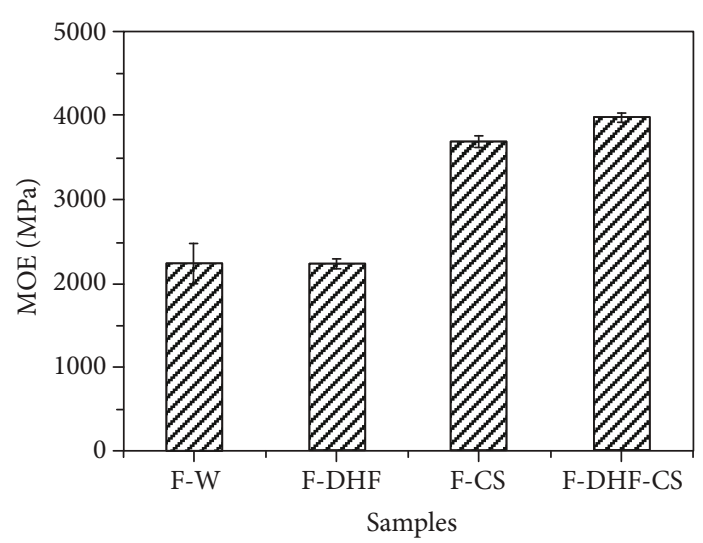

(b)

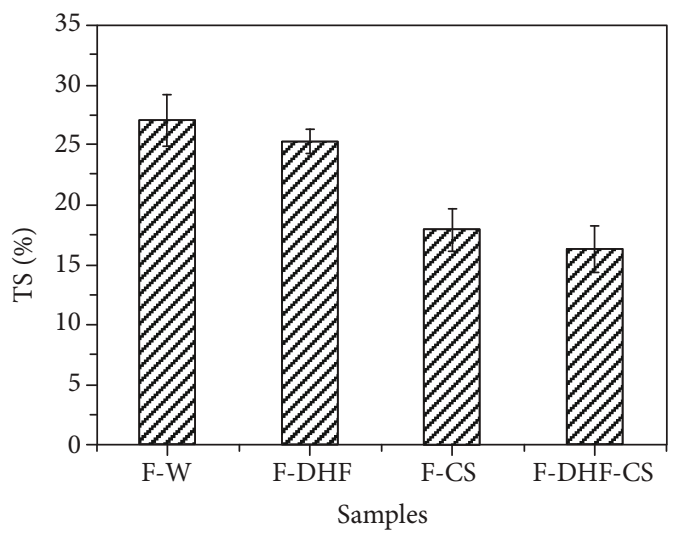

(d)

FIgUre 5: Physical and mechanical properties of F-W, F-DHF, F-CS, and F-DHF-CS: (a) MOR; (b) MOE; (c) IB; (d) TS.

linkages was stronger in F-DHF-CS. This further suggested the amide linkages between cross-linked chitosan and wood fibers. The characteristic peak of the $-\mathrm{OH}$ groups shifted from $3405 \mathrm{~cm}^{-1}$ (F-W) to $3412 \mathrm{~cm}^{-1}$ (F-DHF-CS), which indicated that more hydrogen bonds existed in F-DHF-CS. These hydrogen bonds may result from the interactions between the $-\mathrm{OH}$ groups of wood fibers and the $-\mathrm{OH} /$ amine groups of cross-linked chitosan. According to above FTIR analysis, the amide linkages and hydrogen bonds between wood fibers and cross-linked chitosan were beneficial to the improvement of physical and mechanical properties of wood-based fiberboards.

Figure 5 shows the MOR, MOE, IB, and TS values of FW, F-DHF, F-CS, and F-DHF-CS. As can be seen from Figure 5(a), the MOR values of F-W and F-DHF were similar. The MOR values of F-CS and F-DHF-CS increased to 38.7 $\mathrm{MPa}$ and 42.1 MPa after CS and DHF cross-linked CS were introduced. As shown in Figure 5(b), the MOE values of F$\mathrm{W}$ and F-DHF just reached to $2242.2 \mathrm{MPa}$ and $2238.0 \mathrm{MPa}$. The MOE values of F-CS and F-DHF-CS increased to 3690.7 MPa and 3986.0 MPa. As shown in Figure 5(c), the IB strengths of F-CS and F-DHF-CS were noticeably higher than those of F-W and F-DHF. The F-DHF-CS possessed the highest IB strength values (1.42 MPa). The improvements of F-DHF-CS mechanical properties could result from the amide linkages and hydrogen bonds between wood fibers and cross-linked chitosan. Furthermore, as can be seen from Figure 5(d), the dimensional stabilities of the prepared woodbased fiberboards were also improved using CS and crosslinked CS as the binder. The F-DHF-CS had minimum TS value $(16.3 \%)$. The improvement of dimensional stabilities of F-DHF-CS could be due to the bonding effect of chitosan leading to a tight structure, which decreased the channels for water absorption. This is just like the dense structure of F-DHF-CS observed in SEM.

\section{Conclusion}

In this study, a high-performance wood-based fiberboard with high strength and water resistance which bonded by DHF cross-linked CS was successfully fabricated via hotpressing process. The addition of cross-linked CS improved the mechanical properties and dimensional stability of wood-based fiberboards. The results indicated that F-DHFCS could achieve MOR of $42.1 \mathrm{MPa}, \mathrm{MOE}$ of $3986.0 \mathrm{MPa}$, IB of $1.4 \mathrm{MPa}$, and TS of $16.3 \%$, respectively. FTIR and XPS results show the amide linkages and hydrogen bonds between wood fibers and cross-linked chitosan improved the physical and mechanical properties of wood-based fiberboards. Such high-performance wood-based fiberboard with high strength and dimensional stability is expected to be a promising candidate for green decorative materials. 


\section{Data Availability}

The data used to support the findings of this study are included within the supplementary information files.

\section{Conflicts of Interest}

The authors declare that they have no conflicts of interest.

\section{Authors' Contributions}

Erzhuo Huang and Yanwei Cao contributed equally to this work.

\section{Acknowledgments}

This research was supported by the Open Research Fund of National Forestry and Grassland Administration Key Laboratory of Plant Fiber Functional Materials (Grant No. 2019KFJJ05) and the Scientific Research Foundation of Zhejiang A and F University (2020FR031).

\section{Supplementary Materials}

Figure S1: cross-section SEM images: (a) F-W; (b) F-DHF; (c) F-CS; (d) F-DHF-CS. Figure S2: XPS spectra of F-W, crosslinked CS, and F-DHF-CS. (Supplementary Materials)

\section{References}

[1] W. Gul, H. Alrobei, S. R. A. Shah, and A. Khan, "Effect of iron oxide nanoparticles on the physical properties of medium density fiberboard," Polymers, vol. 12, no. 12, p. 2911, 2020.

[2] A. Pizzi, "Recent developments in eco-efficient bio-based adhesives for wood bonding: opportunities and issues," Journal of Adhesion Science and Technology, vol. 20, no. 8, pp. 829-846, 2006.

[3] R. Tupciauskas, J. Gravitis, J. Abolins et al., "Utilization of lignin powder for manufacturing self-binding HDF," Holzforschung, vol. 71, no. 7-8, pp. 555-561, 2017.

[4] Y. Zhang, J.-Q. Wu, H. Li, T.-Q. Yuan, Y.-Y. Wang, and R.C. Sun, "Heat treatment of industrial alkaline lignin and its potential application as an adhesive for green wood-lignin composites," ACS Sustainable Chemistry \& Engineering, vol. 5, no. 8, pp. 7269-7277, 2017.

[5] X. Li, Y. Li, Z. Zhong et al., "Mechanical and water soaking properties of medium density fiberboard with wood fiber and soybean protein adhesive," Bioresource Technology, vol. 100, no. 14, pp. 3556-3562, 2009.

[6] J. Li, H. Lin, S. R. Bean, X. S. Sun, and D. Wang, "Evaluation of adhesive performance of a mixture of soy, sorghum and canola proteins," Industrial Crops and Products, vol. 157, p. 112898, 2020.

[7] Y. Zeng, P. Xu, W. Yang et al., "Soy protein-based adhesive with superior bonding strength and water resistance by designing densely crosslinking networks," European Polymer Journal, vol. 142, p. 110128, 2021.

[8] C. I. K. Diop, M. Tajvidi, M. A. Bilodeau, D. W. Bousfield, and J. F. Hunt, "Isolation of lignocellulose nanofibrils (LCNF) and application as adhesive replacement in wood composites: example of fiberboard," Cellulose, vol. 24, no. 7, pp. 30373050, 2017.

[9] A. Moslemi, M. Zolfagharlou koohi, T. Behzad, and A. Pizzi, "Addition of cellulose nanofibers extracted from rice straw to urea formaldehyde resin; effect on the adhesive characteristics and medium density fiberboard properties," International Journal of Adhesion and Adhesives, vol. 99, p. 102582, 2020.

[10] Y. Monroy, S. Rivero, and M. A. García, "Sustainable panels design based on modified cassava starch bioadhesives and wood processing byproducts," Industrial Crops and Products, vol. 137, pp. 171-179, 2019.

[11] Y. Zhang, L. Ding, J. Gu, H. Tan, and L. Zhu, "Preparation and properties of a starch-based wood adhesive with high bonding strength and water resistance," Carbohydrate Polymers, vol. 115, pp. 32-37, 2015.

[12] P. S. Bakshi, D. Selvakumar, K. Kadirvelu, and N. S. Kumar, "Chitosan as an environment friendly biomaterial - a review on recent modifications and applications," International Journal of Biological Macromolecules, vol. 150, pp. 1072-1083, 2020.

[13] A. Muxika, A. Etxabide, J. Uranga, P. Guerrero, and K. de la Caba, "Chitosan as a bioactive polymer: processing, properties and applications," International Journal of Biological Macromolecules, vol. 105, no. 2, pp. 1358-1368, 2017.

[14] A. Verlee, S. Mincke, and C. V. Stevens, "Recent developments in antibacterial and antifungal chitosan and its derivatives," Carbohydrate Polymers, vol. 164, pp. 268-283, 2017.

[15] P. Sahariah and M. Masson, "Antimicrobial chitosan and chitosan derivatives: a review of the structure-activity relationship," Biomacromolecules, vol. 18, no. 11, pp. 3846-3868, 2017.

[16] N. Mati-Baouche, P.-H. Elchinger, H. de Baynast, G. Pierre, C. Delattre, and P. Michaud, "Chitosan as an adhesive," European Polymer Journal, vol. 60, pp. 198-212, 2014.

[17] X. Du, Y. Liu, H. Yan et al., "Anti-infective and pro-coagulant chitosan-based hydrogel tissue adhesive for sutureless wound closure," Biomacromolecules, vol. 21, no. 3, pp. 1243-1253, 2020.

[18] A. K. Patel, P. Michaud, E. Petit, H. de Baynast, M. Grédiac, and J.-D. Mathias, "Development of a chitosan-based adhesive. Application to wood bonding," Journal of Applied Polymer Science, vol. 127, no. 6, pp. 5014-5021, 2013.

[19] X. Ji, B. Li, B. Yuan, and M. Guo, "Preparation and characterizations of a chitosan-based medium-density fiberboard adhesive with high bonding strength and water resistance," Carbohydrate Polymers, vol. 176, pp. 273-280, 2017.

[20] X. Ji and M. Guo, "Preparation and properties of a chitosanlignin wood adhesive," International Journal of Adhesion and Adhesives, vol. 82, pp. 8-13, 2018.

[21] Z. Wang, Y. Yan, X. Shen et al., "Lignocellulose-chitosan-multiwalled carbon nanotube composites with improved mechanical strength, dimensional stability and fire retardancy," Polymers, vol. 10, no. 3, p. 341, 2018.

[22] E. de Souza Costa-Junior, M. M. Pereira, and H. S. Mansur, "Properties and biocompatibility of chitosan films modified by blending with PVA and chemically crosslinked," Journal of Materials Science. Materials in Medicine, vol. 20, no. 2, pp. 553-561, 2009.

[23] J. Feng, H. Yi, Z. Lei et al., "A three-dimensional crosslinked chitosan sulfate network binder for high-performance Li-S batteries," Journal of Energy Chemistry, vol. 56, pp. 171-178, 2021. 
[24] J. Lu, P. Jiang, Z. Chen, L. Li, and Y. Huang, "Flame retardancy, thermal stability, and hygroscopicity of wood materials modified with melamine and amino trimethylene phosphonic acid," Construction and Building Materials, vol. 267, p. 121042, 2021.

[25] L. Wu, Y. Chen, Y. Li, Q. Meng, and T. Duan, "Functionally integrated g- $\mathrm{C}_{3} \mathrm{~N}_{4} @$ wood-derived carbon with an orderly interconnected porous structure," Applied Surface Science, vol. 540, p. 148440, 2021.

[26] S. Zhuang, K. Zhu, and J. Wang, "Fibrous chitosan/cellulose composite as an efficient adsorbent for Co(II) removal," Journal of Cleaner Production, vol. 285, p. 124911, 2021.

[27] N. K. Francis, H. S. Pawar, S. Dhara, A. Mitra, and A. Mitra, "Radiopaque hemocompatible ruminant-sourced gut material with antimicrobial physiognomies for biomedical applications in diabetics," ACS Omega, vol. 2, no. 3, pp. 755-764, 2017.

[28] P. Ghosh, A. P. Rameshbabu, N. Dogra, and S. Dhara, "2,5Dimethoxy 2,5-dihydrofuran crosslinked chitosan fibers enhance bone regeneration in rabbit femur defects," RSC Advances, vol. 4, no. 37, pp. 19516-19524, 2014.

[29] M. Li, J. Liu, Y. Hu, X. Gao, Q. Yuan, and F. Zhao, "Investigation of the specularite/chlorite separation using chitosan as a novel depressant by direct flotation," Carbohydrate Polymers, vol. 240, p. 116334, 2020.

[30] W. Zhang, Q. Li, Q. Mao, and G. He, "Cross-linked chitosan microspheres: an efficient and eco-friendly adsorbent for iodide removal from waste water," Carbohydrate Polymers, vol. 209, pp. 215-222, 2019. 